\title{
A Cross-Cultural Analysis of Physician Management of Obesity. Comparing the US, France, Israel and Japan: Little Interest and Little Success
}

Richard J Schuster ${ }^{1^{*}}$, Colleen O’Brien Cherry ${ }^{1}$, Shira Zelbar-Sagi ${ }^{2}$, Hanny Yeshua ${ }^{3}$, Andre Matalon ${ }^{3}$, Olivier Steichen $^{4}$, Didier Duhot ${ }^{5}$, Akira Fujiyoshi $^{6}$ and Katsuyuki Miura ${ }^{6}$

${ }^{1}$ Center for Global Health, University of Georgia, USA

${ }^{2}$ School of Public Health, University of Haifa, Israel

${ }^{3}$ Clalit Israel Health Services, Israel

${ }^{4}$ Hospital Tenon, University of Paris, VI, France

${ }^{5}$ French Society of General Medicine, France

${ }^{6}$ Shiga University of Medical Sciences, Japan

*Corresponding author: Richard J Schuster, MD, MMM, Center for Global Health, University of Georgia, 100 Foster Road, Athens, Georgia 30602, Tel: 706-542-5742; E-mail: rschuster@aol.com

Rec date: Mar 29, 2014, Acc date: July 26, 2014; Pub date: July 30, 2014

Copyright: (c) 2014 Schuster RJ, et al. This is an open-access article distributed under the terms of the Creative Commons Attribution License, which permits unrestricted use, distribution, and reproduction in any medium, provided the original author and source are credited.

\begin{abstract}
Background: Obesity is a global health problem. Physicians are frequently engaged with overweight and obese patients. Obesity guidelines have been successfully implemented on a small scale, but generally physicians struggle to manage obesity effectively.
\end{abstract}

Methods: In a web-based survey, primary care physicians in the United States (US), France, Israel and Japan, were asked how they manage cardiovascular risk factors. They were specifically asked how frequently they saw patients in follow-up for hypertension, hyperlipidemia and obesity.

Results: Respondents (956) included 656 French, 198 Israeli, 45 Japanese, and 53 US physicians. Follow-up for obesity no sooner than 3 months was recommended, by $73 \%$ (US) and $79 \%$ (Israeli) physicians, whereas $67 \%$ of French and $66 \%$ of Japanese physicians recommended more frequent follow-up (3 months or less). Hypertension and hyperlipidemia was managed more aggressively, especially the US, Israel and Japan.

Discussion: Obesity is an international concern, with rates increasing everywhere. The prevalence of obesity is high in the US and Israel and much lower in France and Japan. Chronic disease management is most effective with frequent follow-up. US obesity guidelines recommend frequent (often monthly) follow-up visits. US and Israeli physicians do not appear to be aggressive in managing obesity, whereas French and Japanese physicians report more effort to manage obesity. In the US, obesity management is not considered effective and physicians are uncomfortable attempting to manage obesity with their patients. In France especially, physicians have a more social relationship with their patients and seem oriented toward chronic disease management, including greater attention to lifestyle change.

Conclusion: Obesity has been medicalized and is a profound problem internationally. The medical care system must address obesity management more effectively. Further studies are needed to understand how physicians manage obesity and new approaches should be promoted to improve the outcomes of obesity management.

Keywords: Obesity; Physician practice management; Health systems; Global health; Cardiovascular disease risk Factors; Prevention; Health services research

\section{Introduction}

Obesity is a worldwide problem and one that is increasing in prevalence with serious consequences for the population. Although it is most evident in high income countries, it is a growing concern in middle and low income countries. In the United States, the prevalence of obesity is $34 \%$ of the population; in France $11 \%$, Israel it is $23 \%$, and Japan, $3 \%[1,2]$. Although most experts believe that a population level approach is most important in reducing obesity, primary care physicians are confronted with obesity in their practice of medicine on a frequent basis. Therefore looking at physician practices in obesity management at the individual patient level is important for assessing effective ways to prevent and manage obesity.

In 1999, the US National Institutes of Health (NIH) created practice guidelines for obesity management by health care providers, including physicians and shortly after that a "Practical Guide" to encourage implementation of the guidelines [3,4]. Since their release, there has been little measurement of their implementation and little evidence of their success [5]. Physicians have viewed treatment of obesity at the medical care level to be futile and, in fact, many are embarrassed in even telling patients they are obese [6].

We have previously investigated primary care physician attitudes toward obesity management in the United States and have 
Citation: Schuster RJ, Cherry CB, Zelbar-Sagi S, Yeshua H, Matalon A et al (2014) A Cross-Cultural Analysis of Physician Management of Obesity. Comparing the US, France, Israel and Japan: Little Interest and Little Success. Primary Health Care 4: 166. doi: 10.4172/2167-1079.1000166

Page 2 of 5

demonstrated success in implementing guidelines for obesity treatment via the use of Clinical Engagement and Education Sessions also known as academic detailing [7]. With the prevalence of obesity or overweight patients comprising $75 \%$ of the patients in a clinical practice, it is particularly concerning that only $25 \%$ of patients are diagnosed as obese and only one third of those patients are given goals for weight loss [8]. How do physicians in various countries actually manage obesity? What effort do they commit to this process?

We now report physician clinical management of obesity at an international level. We surveyed primary care physicians from four high income countries: the US, France, Israel and Japan. In an effort to understand how they manage obesity and overweight, we asked them to report on the frequency with which they have patients return for follow-up of Cardiovascular Disease (CVD) risk factor management, including specifically the frequency of follow-up visits for the management of obesity. We have previously reported on other aspects of CVD management from the US, Japanese, and French physicians, but not obesity management [9-11]. We report on the difference in obesity management from that of other CVD risk factors including hypertension - High Blood Pressure (HBP) and hyperlipidemia. Culture has a significant relationship to obesity, yet the influence of culture on health care delivery has not been studied much [12]. We discuss differences in obesity management within a cultural context including country differences in healthcare systems and the physicianpatient relationship.

The understanding of the management of obesity by physicians requires a brief comment on the health systems that provide clinical care to populations in the countries studied. Universal health care is provided in France, Israel, and Japan; it is not currently provided in the US. None of these countries have truly socialized health care systems, but rather insurance "intermediaries" are present in each country. Primary care physicians are self-employed in the Japan and France, often in the US, and not commonly in Israel, where they are typically salaried. Likewise, physicians are paid fee for service in France and Japan, sometimes in the US, and generally not in Israel $[13,14]$. A fee for service payment mechanism has generally been viewed as a mechanism to incentivize return office visits. It could therefore be expected that primary care physicians in France, Japan, and often in the US would see patients with obesity relatively frequently and for relatively short visits, whereas Israeli physicians might see patients less frequently.

\section{Methods}

\section{Survey design}

Primary care physicians (primarily family physicians) from the US, France, Israel, and Japan were surveyed in via an anonymous process. French physicians were surveyed through the French Society of General Medicine, a key organization of family physicians in France. Most US physicians surveyed were members of the Ohio Academy of Family Practice, although primary care physicians in other US states were also included. Physicians in Japan were surveyed through the Shiga Medical Society and physicians in Israel surveyed through the Israeli Society of Family Physicians. The response was from a convenience sample with the organizations' size varying from the Shiga Medical Society having 784 members in private practice to the French Society of General Medicine where approximately 8200 members and affiliates were contacted. A brief multiple choice survey (44-45 questions) was made available through the internet. The survey was written in English and translated into French, Japanese, and Hebrew for the specific country involved. The survey was translated by native speaking co-authors in French, Japanese, and Hebrew and was then back-translated into English from each of the translated versions to confirm its intended wording. Physicians were asked various demographic questions. The survey asked each physician about their practice behaviors regarding CVD risk factor management, such as how often they typically see a patient with hypertension and hyperlipidemia for on-going management (e.g. every 2 months, every 6 months) and how much time they typically spend with a patient with one of these problems (e.g. 5 minutes, 15 minutes). It asked what factors influenced their selection of medication in treating patients with risk factors for cardiovascular disease and what their primary focus was while caring for these patients (lifestyle modification, medication or both), if they routinely provided patient education, if they provided patient education materials, if they had easily accessible information on current guidelines that allowed them real time access during a patient encounter, if they routinely followed guidelines or if they were just aware of them more generally. This study was approved by the IRB of both Wright State University, the University of Georgia, and IRB's in Japan, and Israel. Participants provided informed consent by participating in the study. Some results of this work have been previously published.

\section{Statistical analysis}

The data was collated and the responses of the physicians were compared by country, the type of healthcare system, and CVD risk factor. Data is reported as descriptive statistics of the per cent of physicians in each country who practice specific management practices.

\section{Results}

A total of 933 physicians participated in the survey, with 637 being French, 192 Israeli, 51 Japanese, and 53 US (Table 1).

\begin{tabular}{|c|c|c|c|c|c|}
\hline & & $\begin{array}{l}\text { France } \\
\%(n)\end{array}$ & $\begin{array}{l}\text { U.S \% } \\
\text { (n) }\end{array}$ & $\begin{array}{l}\text { Japan } \\
\%(n)\end{array}$ & $\begin{array}{l}\text { Israel } \\
\%(n)\end{array}$ \\
\hline \multirow[t]{5}{*}{ Age (Years) } & $<35$ & $\begin{array}{l}10 \% \\
(63)\end{array}$ & $\begin{array}{l}13 \% \\
(7)\end{array}$ & $0 \%(0)$ & $\begin{array}{l}7 \% \\
\text { (13) }\end{array}$ \\
\hline & $35-44$ & $\begin{array}{l}12 \% \\
(76)\end{array}$ & $\begin{array}{l}26 \% \\
(14)\end{array}$ & $9 \%(4)$ & $\begin{array}{l}29 \% \\
(57)\end{array}$ \\
\hline & $45-54$ & $\begin{array}{l}47 \% \\
(300)\end{array}$ & $\begin{array}{l}25 \% \\
(13)\end{array}$ & $\begin{array}{l}33 \% \\
(15)\end{array}$ & $\begin{array}{l}25 \% \\
(49)\end{array}$ \\
\hline & $55-64$ & $\begin{array}{l}29 \% \\
(185)\end{array}$ & $\begin{array}{l}21 \% \\
(11)\end{array}$ & $\begin{array}{l}36 \% \\
(16)\end{array}$ & $\begin{array}{l}30 \% \\
(59)\end{array}$ \\
\hline & $>64$ & $2 \%(12)$ & $\begin{array}{l}15 \% \\
(8)\end{array}$ & $\begin{array}{l}22 \% \\
(10)\end{array}$ & $\begin{array}{l}9 \% \\
(18)\end{array}$ \\
\hline \multirow[t]{5}{*}{ Specialty } & $\begin{array}{l}\text { Family/General } \\
\text { Practice }\end{array}$ & $\begin{array}{l}97 \% \\
(620)\end{array}$ & $\begin{array}{l}100 \% \\
(53)\end{array}$ & $\begin{array}{l}35 \% \\
(18)\end{array}$ & $\begin{array}{l}95 \% \\
(183)\end{array}$ \\
\hline & Internal Medicine & $<1 \%(1)$ & 0 & $\begin{array}{l}25 \% \\
(13)\end{array}$ & $\begin{array}{l}<1 \% \\
(1)\end{array}$ \\
\hline & Cardiology & 0 & 0 & $\begin{array}{l}16 \% \\
(8)\end{array}$ & 0 \\
\hline & Nephrology & 0 & 0 & 0 & 0 \\
\hline & Endocrinology & 0 & 0 & $2 \%(1)$ & $4 \%(8)$ \\
\hline
\end{tabular}


Citation: Schuster RJ, Cherry CB, Zelbar-Sagi S, Yeshua H, Matalon A et al (2014) A Cross-Cultural Analysis of Physician Management of Obesity. Comparing the US, France, Israel and Japan: Little Interest and Little Success. Primary Health Care 4: 166. doi: 10.4172/2167-1079.1000166

Page 3 of 5

\begin{tabular}{|l|l|l|l|l|l|}
\hline & Others & $3 \%(16)$ & 0 & $\begin{array}{l}22 \% \\
(11)\end{array}$ & 0 \\
\hline & Total (n) & 637 & 54 & 51 & 192 \\
\hline $\begin{array}{l}\text { Patients Seen per } \\
\text { Week }\end{array}$ & $<25$ & $2 \%(16)$ & $6 \%(3)$ & $0 \%(0)$ & $3 \%(6)$ \\
\hline & $25-74$ & $\begin{array}{l}20 \% \\
(128)\end{array}$ & $\begin{array}{l}30 \% \\
(16)\end{array}$ & $7 \%(3)$ & $\begin{array}{l}18 \% \\
(36)\end{array}$ \\
\hline & $75-124$ & $\begin{array}{l}52 \% \\
(338)\end{array}$ & $\begin{array}{l}43 \% \\
(23)\end{array}$ & $\begin{array}{l}24 \% \\
(11)\end{array}$ & $\begin{array}{l}29 \% \\
(57)\end{array}$ \\
\hline & $125-199$ & $\begin{array}{l}23 \% \\
(151)\end{array}$ & $\begin{array}{l}19 \% \\
(10)\end{array}$ & $\begin{array}{l}33 \% \\
(15)\end{array}$ & $\begin{array}{l}30 \% \\
(59)\end{array}$ \\
\hline & $>199$ & $3 \%(17)$ & $2 \%(1)$ & $\begin{array}{l}36 \% \\
(16)\end{array}$ & $\begin{array}{l}19 \% \\
(38)\end{array}$ \\
\hline
\end{tabular}

\begin{tabular}{|c|c|c|c|c|c|c|}
\hline France & $0(2)$ & $1(10)$ & $30(210)$ & $58(402)$ & $10(71)$ & \\
\hline & $<2 \mathrm{wks}$ & 2 wks-1 mo & $1-3 \mathrm{mo}$ & $3-6 \mathrm{mo}$ & $>6 \mathrm{mo}$ & \\
\hline US & $2(1)$ & $4(2)$ & $21(11)$ & $43(22)$ & $30(16)$ & \multirow{4}{*}{ Obesity } \\
\hline Israel & 1 (2) & $2(4)$ & $18(35)$ & $39(76)$ & $40(78)$ & \\
\hline Japan & $4(2)$ & $17(8)$ & $45(21)$ & $23(11)$ & $11(5)$ & \\
\hline \multirow[t]{2}{*}{ France } & $2(13)$ & $18(125)$ & $47(326)$ & $25(172)$ & $9(63)$ & \\
\hline & \multicolumn{3}{|c|}{ Reported as percentages (count) } & & & \\
\hline
\end{tabular}

Table 2: Frequency of Patient Follow-up Visits for CVD Risk Factors

Among the four countries, obesity is the least prevalent in France and Japan. Yet, the physicians in France and Japan see their patients for follow-up visits for obesity frequently. Seventy one percent of French physicians and $66 \%$ of Japanese physicians see their obese or overweight patients in three months or less, versus $27 \%$ of US physicians and $21 \%$ for Israeli physicians.

Most were between the ages of 45 and 64 years old, with French physicians being somewhat younger and Japanese physicians somewhat older. The vast majority in each country were Family/ General Practice physicians. Japanese physicians see the most patients per week, while French and US physicians see the fewest. Physicians were asked in separate questions about their approach to management of various cardiovascular risk factors, specifically how they managed hypertension, hyperlipidemia, and obesity.

One key measurement of this management was frequency of return visits. There is a notable difference among the countries in the frequency of follow-up visits for any CVD risk factor with Japanese physicians seeing their patients most frequently and American and Israeli physicians least frequently. In all cases however, in each country, no matter what the absolute return visit frequency was, follow-ups for obesity management were less frequent than any of the other CVD risk factors (Table 2). Obesity return visits are recommended by the physicians responding to the survey at longer time intervals than lipid or HBP return visits. (Figures 1 and 2) NIH guidelines recommend monthly visits for at least the first 6 months of engagement in weight loss [4].

High blood pressure is managed with $77 \%$ of all physicians recommending follow-up in three months or less. (Figure 3) Fortythree percent of physicians in this study recommended follow-up for lipid patients who were stable in the 3 to 6 months. Major guidelines recommend follow-up for stable patients with hyperlipidemia at 4 to 6 months range [15].

\begin{tabular}{|c|c|c|c|c|c|c|}
\hline & $<2$ wks & $2 \mathrm{wks}-1 \mathrm{mo}$ & $1-3 \mathrm{mo}$ & $3-6 \mathrm{mo}$ & $>6 \mathrm{mo}$ & \\
\hline US & 2 (1) & $17(9)$ & 35 (18) & $42(22)$ & $4(2)$ & \multirow{4}{*}{ HBP } \\
\hline Israel & $9(18)$ & $27(52)$ & $39(75)$ & $22(43)$ & $3(5)$ & \\
\hline Japan & $2(1)$ & $63(30)$ & 35 (17) & 0 & 0 & \\
\hline \multirow[t]{2}{*}{ France } & $1(7)$ & $9(60)$ & $68(467)$ & $22(153)$ & $0(3)$ & \\
\hline & $<2$ wks & 2 wks-1 mo & $1-3 \mathrm{mo}$ & 3-6 mo & $>6 \mathrm{mo}$ & \\
\hline US & 0 & 0 & $17(9)$ & 66 (34) & $17(9)$ & \multirow{3}{*}{ Lipid } \\
\hline Israel & $1(2)$ & $5(10)$ & $37(72)$ & $46(90)$ & $11(21)$ & \\
\hline Japan & $2(1)$ & $44(21)$ & $50(24)$ & $4(2)$ & 0 & \\
\hline
\end{tabular}

\section{Discussion and Limitations}

Obesity is a profoundly complex problem that is increasing in prevalence globally. The medical care system can and should play a role in obesity management.

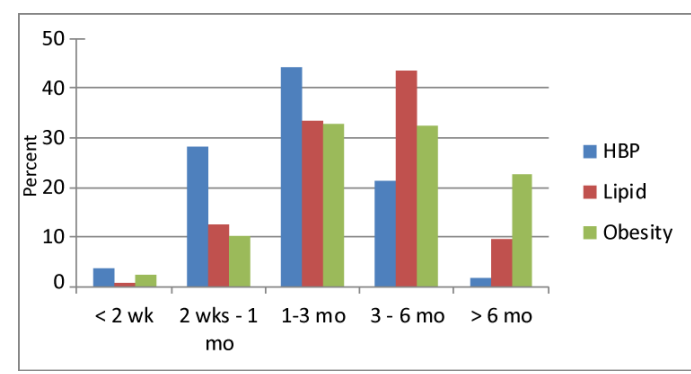

Figure 1: Four nation average return visit frequency: Management of HPB, Lipids, and obesity

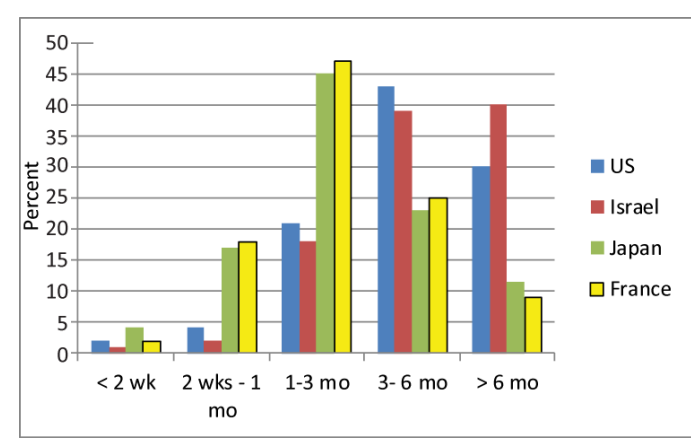

Figure 2: Return visit frequency - Obesity

Most initiatives for obesity management have been done from a population or public health perspective, with a preventive focus on lifestyle and public education. Although these approaches are critically important, they often ignore the medical care community in each 
Citation: Schuster RJ, Cherry CB, Zelbar-Sagi S, Yeshua H, Matalon A et al (2014) A Cross-Cultural Analysis of Physician Management of Obesity. Comparing the US, France, Israel and Japan: Little Interest and Little Success. Primary Health Care 4: 166. doi: 10.4172/2167-1079.1000166

Page 4 of 5

nation, which could play a substantial role in obesity management. In some of our previous work we have identified that physicians do not diagnose and do not treat obesity, particularly obesity that is not morbid obesity [8]. In further investigation we have reported that physicians are uncomfortable discussing obesity with their patients [6]. It might be reasoned then, that they would delay follow-up visits for obesity management beyond the period of time where there would be much hope for effective intervention. In this study, it appears that physicians in all four countries see patients with obesity less frequently in follow-up than they do for the management of other CVD risk factors. High blood pressure, in contrast, is managed aggressively with $77 \%$ of all physicians recommending follow-up in three months or less. (Figure 3) Major guidelines recommend follow-up for stable patients with hyperlipidemia at 4 to 6 months; physicians in this report were consistent with that, as $43 \%$ of physicians recommended followup for stable hyperlipidemic patients in 3 to 6 months [15]

In both France and Japan where obesity prevalence is lower, there appears to be a more aggressive approach to obesity management by the medical care system with more frequent visits. Obesity may receive more attention from physicians as they might perceive of it as more unusual and therefore more deserving of attention.

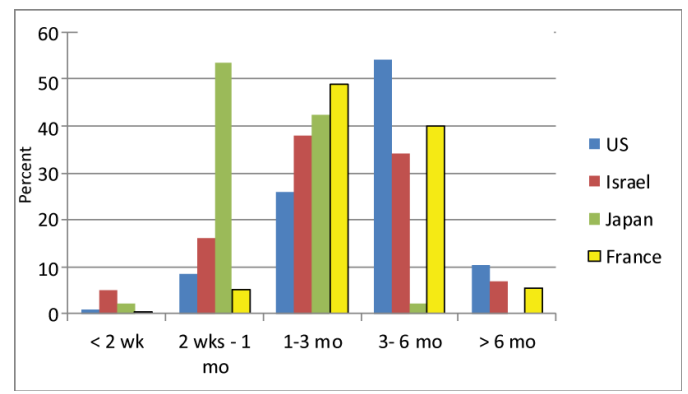

Figure 3: Return visit frequency - HPB and Hyperlipidemia

In France, specifically, not only is the national culture oriented away from obesity but the physician culture is a more social and interactive practice style between physicians and patients and one that focuses more physician energy on lifestyle management instead of medication management of CVD risk factors including obesity [16]. In France there is a greater continuity of care for patients and the trust that this builds between physicians and patients may encourage doctors to discuss uncomfortable topics and/or lifestyle behavior changes that they notice because of their long-term relationship with patients [10]. Additionally, French physicians may be more alarmed with seeing an obese patient because it is less common than in the US, for example. In the US on the other hand, patients often switch physicians more frequently due to a change in healthcare coverage. As chronic disease management requires frequent and relatively intensive and consistent efforts by the healthcare provider the culture of medical practice in France and Japan seem to match this approach more closely than in the US or Israel $[17,11]$. It can be contended that among the CVD risk factors studied, physicians put time and potentially effort into managing obesity. From previous work we know that physicians are uncomfortable discussing obesity with their patients as they once were and discussing death and dying, and sexual dysfunction [6]. It's also possible that physicians view discussing obesity as a nihilistic venture, as many incorrectly believe is the case in tobacco cessation. However, our work has shown that active promotion of obesity diagnosis and treatment guidelines can in fact be quite effective in reducing obesity in a real-world, primary care setting [6]. Further studies are needed to understand how to motivate physicians more fully to manage overweight or obese patients.

Physician payment mechanisms alter physician practice behavior. It is generally assumed that a fee for service payment process encourages frequent visits and a salary based pay for physicians' decreases frequent visits [18]. This might be a partial explanation for the relatively higher visit frequency behavior of French and Japanese physicians and the relatively lower frequency of Israeli physicians. It does not explain the low visit frequency of US physicians. Moreover, French physicians see patients with high-blood pressure less often than US or Israeli physicians which is contrary to the expected behavior in a fee for service incentivized system. It is possible that the "medicalization" of obesity, a relatively recent phenomena, whereby obesity is recognized by physicians, patients, and insurance companies may encourage more frequent and aggressive diagnosis and treatment of obesity in the medical setting [19].

This is a survey conducted of physicians in clinical practice in four countries. Physician statements about their practices do not necessarily correlate fully with observed practices. Samples, especially in the United States and Japan, were small and heterogeneous in size and are not nationally representative. Although we contend that reported follow-up visit frequency is a reasonable estimation for intensity of obesity management, it is proposed as a proxy measure and may not be representative of overall physician obesity management. A larger sample size and a more representative sampling process could demonstrate different outcomes. Future studies should use office visit billing records, percentage of office visit time to directly measure the intensity of obesity management, or direct observation of the physician - patient encounter. A more detailed study is recommended to confirm that should be considered preliminary information.

Finally, although obesity is characterized as a global problem (one that transcends borders), it appears to be managed as a national problem (one that has medical management that varies from nation to nation - influenced by culture, medical care system, financing of health care, or other unidentified factors) [20]. Although the spread of obesity have been well documented, physician management practices have not been noted at a global or even at an international (comparative) level [21]. While individual national or regional culture must be recognized and incorporated into care, efforts should be made for it to be medically managed as a global problem, with emphasis on a consistent, transcultural approach to the management of this dramatically increased health problem.

\section{Conclusion}

In order to effectively treat and prevent obesity, the medical care system must address overweight and obesity at the individual patient care level. Although guidelines have been created and can be successfully implemented, it appears that current physician practices do not address obesity management successfully. Whether physicians are uncomfortable with the discussion of obesity, or perceive their efforts to be nihilistic, physician practices in the management of obesity do not appear to match with desired approaches for chronic disease management [16]. This study demonstrates that in the four high income countries studied, physicians defer follow-up visits for the management of obesity. Interestingly, physicians in France and Japan, 
Citation: Schuster RJ, Cherry CB, Zelbar-Sagi S, Yeshua H, Matalon A et al (2014) A Cross-Cultural Analysis of Physician Management of Obesity. Comparing the US, France, Israel and Japan: Little Interest and Little Success. Primary Health Care 4: 166. doi: 10.4172/2167-1079.1000166

Page 5 of 5

where the prevalence of obesity is much lower, seem to be more aggressive in the management of obesity by the measure that we have studied. French physicians specifically, who are focused more on lifestyle management, appear to be more willing to commit time and their professional energy in their practice to managing obese patients.

It is recommended that physician awareness of obesity management guidelines be promoted and that physicians and other healthcare providers should follow recommended chronic disease management practices by increasing the frequency with which they follow-up their patients who are overweight and obese.

Further studies by using different methods are needed to understand and improve how physicians manage obesity.

\section{Acknowledgement}

The Israeli survey has been funded by Pfizer Israel. The Government of France provided partial funding for the French survey. Gilles Hebbrecht, MD, of the French Society of General Medicine, played a key role in the development and execution of the study. The authors would like to express special appreciation to the many primary care physicians in the France, Israel, Japan, and the United States who took the time to complete this survey.

\section{References}

1. OECD (2013) "Overweight and obesity among adults," in Health at a Glance OECD Indicators, OECD Publishing.

2. Keinan-Boker L, Noyman N, Chinich A, Green M, Nitzan-Kaluski D (2005) Overweight and Obesity Prevalence in Israel: Findings of the First National Health and Nutrition Survey (MABAT). Israel Medical Association Journal 7: 219-223.

3. NHLBI (1998) Clinical Guidelines on the Identification, Evaluation and Treatment of Overweight and Obesity in Adults: The Evidence Report, NIH Publication 98-4083

4. NHLBI Obesity Education Initiative (2000) The Practical Guide: Identification, Evaluation, and Treatment of Overweight and Obesity in Adults. Bethesda, MD: National Institutes of Health, NIH Publication 00-4084.

5. Salinas GD, Glauser TA, Williamson JC, Rao G, Abdolrasulnia M (2011) Primary care physician attitudes and practice patterns in the management of obese adults: results from a national survey. Postgrad Med 123: 214-219.

6. Schuster RJ, Terwoor NA and Tasosa J (2008) Translational Research Implementation of NHLBI Obesity Guidelines in a Primary Care Community Setting: the Physician Obesity Awareness Project. Journal of Nutrition, Health, \& Aging 12: 704S.
7. Schuster RJ, Cherry CO, Smith ML (2013) The clinician engagement and education session: modernizing "academic detailing". Am J Med Qual 28: 533-535.

8. Orlowski, Marietta, Adkins S, Ellison S, Choh A, et al. (2013) Assessment and Management of Adult Obesity in Primary Care Practice," World Medical \& Health Policy 5: 19-36.

9. Schuster RJ, Steichen O, Ogunmoroti O, Ellison S, Terwoord N, et al. (2011) Physician cardiovascular disease risk factor management: practices in France vs the United States. J Clin Hypertens (Greenwich) 13: $10-18$.

10. O'Brien Cherry C, Steichen O, Mathew A, Duhot D, Hebbrecht G, et al. (2012) A culture of care: the French approach to cardiovascular risk factor management. J Am Board Fam Med 25: 477-486.

11. Schuster RJ, Zhu Y, Ogunmoroti O, Terwoord N, Ellison S, et al. (2013) Physician cardiovascular disease risk factor management: practice analysis in Japan versus the USA. Qual Prim Care 21: 51-60.

12. Kirk JK, Hildebrandt C, Davis S, Crandall SJ, Siciliano AB, et al. (2014) Perceptions of culturally competent diabetes management in a primary care practice. J Cult Divers 21: 22-28.

13. Rosen B and Merkur S (2009) Israel: Health system review Health Systems in Transition 11: 1-226.

14. Thomson S, R Osborn, D Squires, M Jun (2012) International Profiles of Health Care Systems. Commonwealth Fund.

15. Third Report of the National Cholesterol Education Program (NCEP) (2002) Expert Panel on Detection, Evaluation, and Treatment of High Blood Cholesterol in Adults (Adult Treatment Panel III) Final Report National Cholesterol Education Program National Heart, Lung, and Blood Institute National Institutes of Health NIH Publication No. 02-5215.

16. Guiliano M (2007) French Women Don't Get Fat. Vantage Books, New York.

17. Steinberg BA, Bhatt DL, Mehta S, Poole-Wilson PA, O'Hagan P, et al. (2008) Nine-year trends in achievement of risk factor goals in the US and European outpatients with cardiovascular disease. Am Heart J 156: 719-727.

18. Eddy DM (1997) Balancing cost and quality in fee-for-service versus managed care. Health Affairs. 16: 162-173.

19. Blackburn G (2011) Medicalizing Obesity: Individual, Economic and Medical Consequences. Virtual Mentor: American Medical Association Journal of Ethics 13: 890-895.

20. Imes CC, Burke LE2 (2014) The Obesity Epidemic: The United States as a Cautionary Tale for the Rest of the World. Curr Epidemiol Rep 1: $82-88$.

21. The Global Burden of Metabolic Risk Factors for Chronic Diseases Collaboration Cardiovascular disease, chronic kidney disease, and diabetes mortality burden of cardiometabolic risk factors from 1980 to 2010 (2004) A comparative risk assessment, Lancet Diabetes Endocrinology. 